Ann. Génét. Sél. anim., I973, 5 (I), I47-I53.

INFORMATIONS

\title{
TENDANCES ACTUELLES DE L'EXPÉRIMENTATION EN MATIÈRE DE SÉLECTION BOVINE
}

\author{
J.-J. COlleAU, F. MÉNISSIER, L. S. MONTEIRO* et B. VISSAC \\ Station de Génétique quantitative et appliquée, \\ Centre national de Recherches zootechniques, I. N. R. A., \\ 78350 Jouy en Josas \\ * Animal Breeding Organization \\ West Main Road, Édimbourg, EH 9, $3 \mathrm{JN}$, Écosse
}

Stimulés par une demande de viande bovine en augmentation continuelle, les sélectionneurs recherchent des solutions nouvelles parmi lesquelles les croisements interraciaux prennent une part importante. On voit, en outre, se développer le souci constant de la rentabilité en même temps que l'on s'efforce de combiner le mieux possible les élevages à viande et à lait.

A la demande de la Rédaction des A. G. S. A. nous voudrions montrer la forme que prennent les projets de recherche consacrés à cette discipline dans trois pays qui sont chacun représentatif d'un type d'évolution de la production bovine : la Grande-Bretagne, les États-Unis et la France (').

\section{I. - EXPÉRIMENTATIONS BRITANNIQUES SUR L'EFFICACITÉ DE LA PRODUCTION BOVINE}

Pour étudier quel rendement ont les animaux dans l'utilisation de leur nourriture pour croître et pour produire du lait, des expériences ont été récemment mises en place sur deux domaines de 1'A. B. R. O. (Animal Breeding Research Organization) : à Blythbank farm dans le Peeblesshire et à Cold Norton farm dans le Staffordshire. Dans les deux cas les animaux sont alimentés ad libitum avec un régime complet standard pour bovin en croissance et en lactation mis au point spécialement par Unilever. Ainsi se trouve garantie, dans des limites raisonnables, la constance de la composition alimentaire pour tous les animaux d'un bout de l'année à l'autre et d'une année sur l'autre. La consommation individuelle est mesurée une fois tous les quinze jours jusqu'au troisième vêlage pour les femelles et jusqu'au départ pour les mâles. La produc-

( ${ }^{1}$ ) L. S. Monteiro a traité des expériences britanniques, B. Vıssac des projets américains et J-J. CoLLEAU et F. MÉnISSIER de l'expérimentation française. 
tion laitière est mesurée une fois par semaine. En plus on prend des mesures linéaires corporelles et on dose le lait.

Le M. M. B. (Milk Marketing Board) et le S. M. M. B. (Scottish Milk Marketing Board) coopèrent pour collecter et conserver de la semence parmi les descendants de ces femelles de toutes les races. Ainsi il sera possible de produire un nombre considérable de types différents de croisés.

En utilisant un schéma de croisement multiple comprenant jusqu'à 8o types d'hybrides avec 4 descendants par type, l'effet général du croisement et du format de la mère sur les différents aspects de l'efficacité productrice pourront être estimés avec une précision raisonnable à partir d'effectifs limités. De même on pourra se faire une idée de la potentialité sélective d'une telle population polycroisée.

L'expérience de Blythbank d'une part met en jeu jusqu'à zo races différentes, chacune étant représentée par 2 à 4 femelles provenant de 2 à 4 taureaux. Ce schéma multiracial fournit, pour les paramètres génétiques intra-race, des estimées de portée plus générale encore que légèrement moins précises que celles obtenues sur une ou deux races. Entre races le schéma procurera une estimation générale de la variation de l'efficacité productive plutôt qu'une comparaison précise entre deux races spécifiées. Toutefois, de tout premier intérêt, seront les études entre races de certains rapports, comme celui de la nourriture ingérée aux taux de croissance et à la production laitière ou celui de la taille corporelle au rendement.

L'expérience de Cold Norton quant à elle est centrée sur une comparaison de deux principales races laitières : la Frisonne et la Jersey. Chaque race sera représentée par environ roo femelles fondatrices achetées très jeunes. Dans chaque race des mâles d'insémination bons et mauvais déjà testés au M. M. B. par la méthode de comparaisons aux contemporaines seront testés sur la descendance. Les différences entre bons et mauvais mâles sur la production laitière des filles en ferme seront comparées aux écarts mesurés expérimentalement sur l'efficacité de la production laitière et celle de la première croissance des descendantes femelles. On examinera également, en collaboration avec le Meat Research Institute, l'efficacité de la production de viande des fils à trois âges d'abattage. En outre, les paramètres intra-race d'efficacité de production seront estimés à partir d'analyses entre demi-sœurs et parents-descendants.

Dans une certaine mesure les deux expériences se complètent. Elles fourniront une quantité considérable d'informations sur la variabilité génétique et sur les corrélations génétiques entre et intra-races. Elles permettront aussi une évaluation des avantages comparés de la sélection et des croisements en terme d'efficacité de l'utilisation des aliments.

\section{II. - LES EXPÉRIMENTATIONS RÉALISÉES AUX U. S. A. SUR LES RACES BOVINES FRANÇAISES PAR LE SERVICE FÉDÉRAL, DE RECHERCHES}

Devant la demande croissante du marché en viande maigre, les expérimentations utilisant la race charolaise, présente aux U. S. A. depuis 1935 environ, se sont développées dans différentes stations expérimentales d'États. Cette race a d'abord été utilisée comme race paternelle en comparaison avec les races à viande d'origine anglo-saxonnes, ou zébus (Louisianne : r96o-r96I ; North Carolina : I968) ; on a mis en évidence son potentiel élevé de croissance musculaire. Paralèllement étaient entrepris au Texas (1966-1967) des essais sur l'utilisation des aliments par le couple mère-veau à partir d'animaux de grand et de petit format appartenant aux races charolaise et Hereford. Enfin depuis 1967, I968 les Universités de plusieurs États (Montana, Missouri, Ohio) ont mis en place des plans factoriels de croisement faisant intervenir les races charolaise, Angus et Hereford, et permettant une analyse plus complète des potentialités génétiques respectives de ces races. 
A la suite de ces premiers essais incluant la race charolaise et mettant en évidence ses avantages et ses inconvénients, il est en fait apparu qu'un inventaire plus complet des races européennes - notamment françaises - et de leurs possibilités d'utilisation pour accroître la production de viande en croisement avec le cheptel autochtone (Angus, Hereford) devait être entrepris. Une telle expérience ne pouvait être réalisée que dans un centre de recherches fédéral. Telle est l'origine du Meat Animal Research Center (M. A. R. C.) dirigé par K. Gregory et loca lisé à Clay Center (Nebraska) sur une base de $\mathbf{1} 8$ ooo ha cédée à l'U. S. D. A. par la Marine américaine. Ce centre doit héberger environ 5 ooo vaches allaitantes ainsi que ro ooo brebis et Ioo à 200 truies sur lesquelles seront réalisées des expériences analogues à celles effectuées sur bovins à viande.

Les expérimentations entreprises à Clay Center, expérimentations dont l'objet essentiel réside dans l'utilisation optimale du matériel biologique (amélioration génétique) pour intensifier la production, diminuer les couts de production et augmenter la qualité du produit commercial (viande), ont en fait un caractère pluridisciplinaire très marqué. Elles rassemblent actuellement des chercheurs généticiens, physiologistes, biochimistes, microbiologistes. Les problèmes d'habitat, de mécanisation dans l'exploitation du fourrage et des animaux, ainsi que les problèmes économiques vont être abordés dans des phases ultérieures.

\section{TABLEAU I}

Schéma des croisements réalisés à Clay Center (M. A. R. C.) :

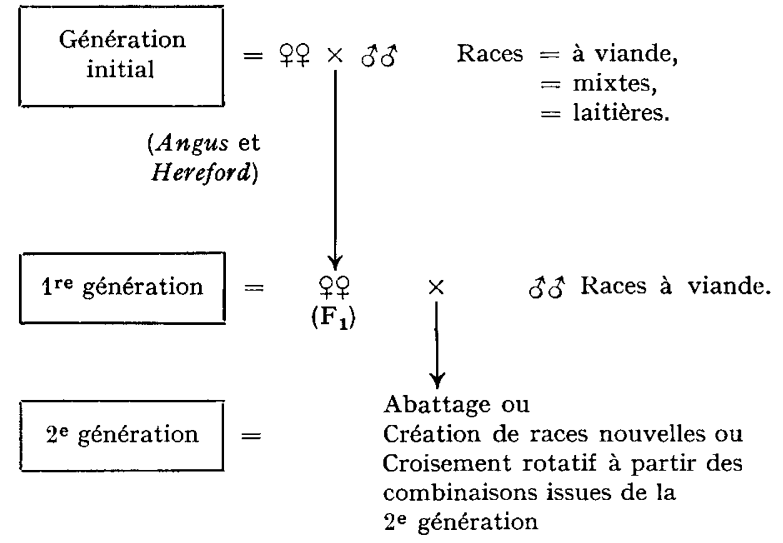

En ce qui concerne les bovins à viande et les ovins, le plan expérimental correspond à une application du travail de Dickerson dans Animal Breeding Abstract (1969) : "Experimental approach in utilizing breed resources. "Il consiste à étudier systématiquement les diverses stratégies d'amélioration des races Angus et Hereford à partir de croisements avec des races à viande européennes, des races mixtes et spécialisées pour la production latière : croisement simple, croisement à double étage, croisements rotatifs et éventuellement de métissage avec création de nouvelles races. Les expérimentations échelonnées sur ro à ${ }_{5} 5$ ans comportent une lignée témoin constituée par des animaux croisés Angus $\times$ Hereford, et sont établies en vue d'analyser l'importance des actions additives et non additives des gènes sur les caractères intéressant l'animal de boucherie d'une part, sur ceux relatifs à la fertilité des femelles et aux qualités maternelles d'autre part.

Deux cycles expérimentaux ont été planifiés à ce jour. Ils comportent chacun l'utilisation de sperme de taureaux de différentes races sur des femelles Angus et Hereford (I re génération). 
Les femelles croisées sont, elles, inséminées avec du sperme des races considérées comme races paternelles en vue d'un croisement commercial ( $2^{e}$ génération). En fait, ceci n'est réalisé qu'à partir du $2^{\mathrm{e}}$ vêlage ; dans cette $2^{\mathrm{e}}$ étape, on utilise sur les génisses des taureaux de races non apparentées dans le but de tester leurs qualités maternelles et de réduire au minimum les difficultés de vêlage (tabl. $\mathbf{r}$ ).

Les races exotiques utilisées dans cette expérimentation et qui sont indiquées dans le tableau 2 correspondent aux races existant actuellement aux États-Unis et les reproducteurs ont été choisis par des sociétés commerciales.

TABLEAU 2

Races européennes expérimentées à Clay Center (M. A. R. C.) sur un cheptel femelle de base Angus-Hereford

\begin{tabular}{|c|c|c|c|}
\hline \multirow{3}{*}{$\mathrm{N}^{\circ}$ de cycle } & \multicolumn{3}{|c|}{ Génération } \\
\hline & \multirow{2}{*}{$1^{\mathrm{re}}$} & \multicolumn{2}{|r|}{$2^{e}$} \\
\hline & & Génisses & Vaches \\
\hline $\begin{array}{c}1^{\mathrm{er}} \\
(1968-1971)\end{array}$ & $\begin{array}{l}\text { Jersey } \\
\text { South Devon } \\
\text { Limousin } \\
\text { Pie Rouge de l'est } \\
\text { Charolais }\end{array}$ & $\begin{array}{l}\text { Brahman } \\
\text { Devon } \\
\text { Holstein }\end{array}$ & $\begin{array}{l}\text { Limousin } \\
\text { Simmental } \\
\text { Charolais }\end{array}$ \\
\hline $\begin{array}{c}2^{\mathrm{e}} \\
(1972-1975)\end{array}$ & $\begin{array}{l}\text { Red Polled } \\
\text { Brown Swiss } \\
\text { Maine-Anjou } \\
\text { Blond d'Aquitaine } \\
\text { Chianina }\end{array}$ & & $\begin{array}{l}\text { Maine-Anjou } \\
\text { Blond d'Aquitaine } \\
\text { Chianina }\end{array}$ \\
\hline
\end{tabular}

Les années indiquées correspondent au début et à la fin des inséminations relatives à la production des veaux de $\mathbf{1}^{\text {re }}$ génération.

Outre les contrôles zootechniques classiques sur les mères et sur les veaux pendant les phases d'élevage et d'engraissement, des recherches sur la physiologie et la nutrition sont réalisées, notamment sur :

- la réponse différentielle des races à des traitements hormonaux (FSH) à doses variées,

- l'incidence du sevrage précoce (5-7 semaines) sur la fertilité ultérieure de la vache et de son veau femelle, - l'incidence du niveau d'énergie avant la mise bas ( 3 niveaux) et après vêlage (3 niveaux) sur les difficultés de vélage et la fertilité au cours du cycle suivant.

\section{III. — LES EXPÉRIMENTATIONS FRANÇAISES SUR L'AMÉLIORATION GÉNÉTIQUE DES BOVINS}

Les méthodes d'utilisation et d'exploitation des femelles bovines conduisent à distinguer, en France, trois situations essentielles :

Io Les races mixtes ou laitières dont les femelles sont soumises à la traite (normande, frisonne, montbéliarde). 
$2^{\circ}$ Les races rustiques à double ou triple aptitude qui, du fait de la suppression de la traite et de la traction animale, sont orientées vers la production de viande en troupeaux spécialisés et soumises au croisement avec des taureaux de races à viande (aubrac, gasconne, par exemple).

$3^{\circ}$ Les races à viande traditionnelles (charolaise, limousine).

Le territoire français se laisse assez bien diviser selon ces critères en trois zones. La première couvre le nord du pays (races mixtes ou laitières), la seconde le sud (races rustiques), la troisième le centre (races à viande, (cf. fig. I).

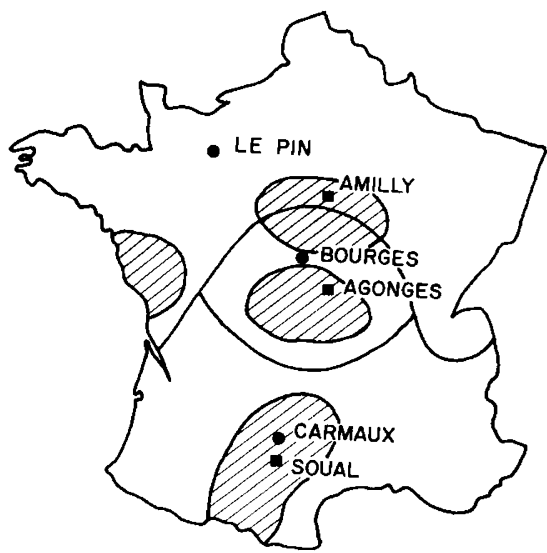

FIG. I. - Les trois zones correspondant aux trois grands types actuels d'exploitation des femelles bovines en France

Zone I (Nord ) : utilisation des femelles pour la traite, Zone 2 (Sud) : utilisation des femelles pour le croisement industriel viande, Zone 3 (Centre) : élevage à viande traditionnel. En hachures on a représenté les aires d'extension des centres d'insémination qui appliquent des programmes de sélection. Les domaines expérimentaux de l'I. N. R. A. sont figurés par des ronds noirs.

Du fait du développement important de l'insémination artificielle les chercheurs de la Station de Génétique quantitative et appliquée de l'I. N. R. A. ont été conduits à étudier et mettre en place des schémas expérimentaux de sélection des géniteurs mâles adaptés à chacune de ces 3 situations, en liaison avec divers organismes techniques responsables de l'insémination et de la sélection. Ces essais pilotes ont été réalisés :

- pour les races mixtes et laitières par la Coopérative d'Élevage du Centre-Nord à Amilly (45-Loiret) ; (Zone I).

- pour les races à viande utilisées en croisement industriel par la Coopérative d'Élevage du SudOuest à Soual (8I-Tarn) ; (Zone 2).

- pour les races à viande dans les zones d'élevage en race pure par l'Association de Testage de la race charolaise à Agonges (o3-Allier) ; (Zone 3).

Parallèlement à ces opérations expérimentales de sélection réalisée sur des populations bovines commerciales, des expériences destinées à accroître l'efficacité et à rendre plus prospectifs les schémas de croisements et de sélection ont été mises en place dans dës domaines expérimentaux de l'I. N. R. A., situés dans les trois zones ci+dessus évoquées : au Pin au-Haras (Zone I : races laitières et mixtes), à Carmaux (Zone 3 : races à viande pour le croisement industriel) et à Bourges (Zone 3 : races à viande pour les zones d'élevage traditionnel).

Tous les animaux de ces troupeaux destinés à l'engraissement (taurillons ou vaches de réforme), sont transportés puis engraissés à l'étable expérimentale de La Minière, près de Jouy en Josas (78). Ils sont enšuite abattus et contrôlés en carcasse aux abattoirs du Centre national de Recherches zotechniques de Jouy en Josas (78-Yvelines) ou du Centre de Recherches zootechniques et vétérinaires de Theix (63-Puy-de-Dôme). 


\section{A. - Le Projet expérimental du Pin-au-Haras sur les races mixtes et spécialisées}

Ce projet concerne un troupeau de $5_{50}$ vaches laitières avec leurs descendants mâles et femelles. Il a débuté en 1967, au Pin-au-Haras en Normandie (6I - Orne) dans une ferme où étaient déjà, effectués depuis io ans de nombreux travaux sur l'engraissement des bovins à l'herbe (Station de Recherches sur l'Élevage des Ruminants, Theix 63 -Puy-de-Dôme). Il a visé, de façon générale à préciser le sens de la sélection des bovins laitiers pour accroître leur valeur globale de production de lait et de viande, à analyser les liaisons entre ces productions ainsi que les mécanismes physiologiques qui les déterminent.

D'une manière plus précise, trois thèmes généraux sont abordés avec ce troupeau. Dans un premier thème, on étudie les répercussions de la sélection laitière sur la production de viande Pour cela, on effectue deux sélections sur la production laitière : l'une montante et l'autre descendante ; on utilise dans ce but le sperme des taureaux testés dans le cadre des programmes de contrôle de descendance réalisés en France. Une seule race est impliquée par ce thème, la Normande. Le second thème vise à analyser l'intérêt possible de mesures physiologiques pour la sélection laitière ou bouchère. L'idée essentielle est de rassembler des génotypes représentant une gamme très variée du point de vue des aptitudes laitière et bouchère, de façon à étudier, entre races, les réponses corrélatives au niveau physiologique. De toute manière, il s'agit là d'une étude préliminaire, la vérification intra-race des liaisons éventuelles entre races s'avérant nécessaire. Les génotypes étudiés sont les suivants: Charolais (type viande), Normand (type mixte), Holstein-Canadien (type laitier) et les croisements Charolais $\times$ Normand et Holstein $\times$ Normand. En troisième lieu, enfin, on étudie l'intérêt économique global (lait et viande) des génotypes impliqués dans l'étude des deux premiers thèmes. Un accent particulier est mis sur la mesure de l'efficacité de la transformation des aliments, en lait ou en gain de poids (vaches laitières), en muscles ou en gras (taurillons, vaches de réforme).

L'étude du premier thème sera particulièrement longue en raison de la durée de l'intervalle de générations ( $\mathrm{I}^{\mathrm{er}}$ vêlage à 3 ans). Les liaisons génétiques intra-races entre les critères de croissance, composition corporelle d'une part, et les critères de production laitière d'autre part, ne seront probablement pas connues avec une précision suffisante en 1980, s'il s'agit de la production de viande par les jeunes bovins et avant r985 s'il s'agit de la production de viande par les vaches de réforme. L'étude du second thème a débuté tout récemment par quelques mesures de taux hormonaux sur un petit échantillon d'animaux. Le troisième thème est actuellement le plus avancé, bien que la comparaison des 5 génotypes ne puisse se faire que sur des critères de croissance et de production de viande. On a fait apparaître une forte interaction génotype $\times$ âge sur la vitesse de croissance, l'âge étant largement confondu avec le régime alimentaire (lait, fourrages grossiers, herbe, fourrages déshydratés). Malgré cela, le croisement Holstein $\times$ Normand semble présenter un intérêt assez constant pour la vitesse de croissance et dans un degré moindre, pour les performances de carcasse et d'engraissement.

\section{B. - La sélection du caractère culard au domaine de Carmaux}

Ce domaine de $\mathbf{I} 20$ ha héberge un troupeau de I 20 femelles présentant le caractère culard. Il est destiné à constituer une souche de bovins présentant ce caractère et destinée à une utilisation des mâles pour un croisement terminal. Ces femelles sont inséminées avec du sperme de mâles culards issus du troupeau ou utilisés dans les centres d'insémination français. Tous les mâles produits à Carmaux sont contrôlés individuellement en station puis, pour ceux retenus, sur descendance croisée dans le cadre des programmes de sélection des taureaux d'insémination de races à viande destinés au croisement industriel. La conduite du troupeau est conçue en vue de minimiser les inconvénients liés au caractère culard chez les femelles :

- déclenchement artificiel de l'œstrus, 
- mise bas par césarienne en général,

- allaitement artificiel des veaux.

Deux cycle expérimentaux sont en cours de réalisation. Le premier concerne les races charolaise et blonde d'Aquitaine (animaux de la génération " 0 " nés en 1967 et 1968); il débute par un plan factoriel de croisement entre bovins culards de ces 2 races. On compte ensuite produire des animaux métis $\left(\mathrm{F}_{2}, \mathrm{~F}_{3} \ldots\right)$ entre les races concernées. L'objectif de sélection de ces métis visera plus précisément à accroître le potentiel de croissance musculaire tout en maintenant un poids et une morphologie à la naissance compatible avec des conditions normales du vêlage en croisement.

Le deuxième cycle expérimental intéresse les races Maine-Anjou et blonde du Sud-Ouest (animaux de la génération " 0 " nés en 1971).

\section{C. - Croisements entre les races à viande françaises à Bourges}

Ce projet qui est en cours de réalisation près de Bourges au domaine de Galle, Avord (1 8 - Cher) a pour but d'analyser les effets additifs et non additifs des gènes intra-race et entre races à viande françaises sur les caractères de croissance, d'efficacité alimentaire et de compositon corporelle d'une part, sur la fertilité, l'aptitude au vêlage et les caractéristiques maternelles d'autre part. On a constitué dans ce but un troupeau de 2 Io femelles nées en $197^{\circ}$ et appartenant aux races charolaise, limousine et Maine-Anjou. Chaque femelle est accouplée successivement trois années consécutives avec du sperme d'un mâle de chacune des 3 races. Les mâles de $\mathrm{I}^{\text {re }}$ génération seront abattus à 2 âges différents ; quant aux femelles obtenues, elles seront accouplées également pendant 3 années en vue d'obtenir des produits de croisement de retour et de croisement 3 races (1975-1979). On dispose, par ailleurs, d'un lot témoin de 25 génisses Hereford conduites en race pure. Tous ces animaux (taurillons et femelles reproductrices) sont exploités en zéro pâturage. 